\title{
The Genetic Analysis of Cystic Fibrosis Patients with Seven Novel Mutations in the CFTR Gene in the Central Anatolian Region of Turkey
}

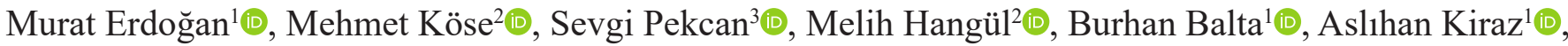 \\ Gizem Akıncı Gönen ${ }^{1}$, Ayşe Gül Zamani ${ }^{1}$, Mahmut Selman Yı1dırım, Tuğba Ramaslı Gürsoy ${ }^{5}$, \\ Fatih Ezgu ${ }^{6}$, Tuğba Şişmanlar Eyüpoğlu ${ }^{5}$, Ayse Tana Aslan ${ }^{5}$
}

\author{
${ }^{1}$ Department of Medical Genetics, Kayseri Training and Research Hospital, Kayseri, Turkey \\ ${ }^{2}$ Division of Pediatric Pulmonology, Department of Pediatrics, Erciyes University, Kayseri, Turkey \\ ${ }^{3}$ Meram Medicine Faculty, Division of Pediatric Pulmonology, Department of Pediatrics, Necmettin Erbakan University, Konya, Turkey \\ ${ }^{4}$ Meram Medicine Faculty, Department of Medical Genetics, Necmettin Erbakan University, Konya, Turkey \\ ${ }^{5}$ Division of Pediatric Pulmonology, Department of Pediatrics, Gazi University, Ankara, Turkey \\ ${ }^{6}$ Department of Pediatric Metabolic Disorders and Pediatric Genetics, Gazi University, Ankara, Turkey
}

Background: Cystic fibrosis, a pulmonary disease which is an autosomal recessive, inherited, multisystemic genetic disease commonly seen in the Caucasian race, is the most frequent cause of mortality and morbidity. So far, more than 2000 disease-causing gene variants have been found and this number has been increasing with the studies conducted. Although there is not yet enough data that include the Turkish population, the recent increase of studies is noteworthy.

Aims: To discover the genetic variation in patients diagnosed with cystic fibrosis in the Central Anatolian region.

Study Design: Cross-sectional study.

Methods: The study was carried out in the Central Anatolian region in 3 pediatric pulmonology departments (Kayseri, Konya, and Ankara) in Turkey between July 2014 and December 2017. The Sanger and Next Generation Sequence analyses were used for exon and exon-intron boundaries in the cystic fibrosis transmembrane conductance regulatory (CFTR) gene, and in selected patients, mutation analysis was performed using the Multiplex Ligation-dependent Probe Amplification technique for large deletions and duplications.

Results: CFTR gene analysis was performed for 316 patients and 215 of them were genetically diagnosed with cystic fibrosis. Sixtythree different variants were defined in these patients and 7 of these were large deletions/duplications detected with the MLPA method. The most frequent variants were F508del (29.6\%), G85E (8.2\%), N1303K (8.2\%), Y515* (7.5\%), and G542* (3.4\%).

Conclusion: Using sequencing and Multiplex Ligation-dependent Probe Amplification methods, the identification of seven new mutations that were not previously reported in the literature contributes to a better understanding of the heterogeneous nature of CFTR mutations in the Turkish population. When no mutations are detected (pathogenic/probably pathogenic) in clinically compatible cases, Multiplex Ligationdependent Probe Amplification analysis contributes significantly to the diagnosis.

\section{INTRODUCTION}

Cystic fibrosis (CF; OMIM \#219700) is the most frequent autosomal recessive inherited genetic disease of the Caucasian race. Its incidence is $1 / 2500-3500$ newborns. ${ }^{1,2}$ It is more frequent in European and European-origin communities when compared with other communities. ${ }^{3}$ Recently, we showed the incidence of CF to be $1 / 3400$ live births in our region, similar to other European communities. ${ }^{4}$ While the percentage of carriers differs according to ethnic origins, it is $1 / 25$ in Northern Europe, $1 / 29$ among Ashkenazi Jews, ${ }^{5,6} 1 / 46$ in Hispanic Americans, and 1/65 in African Americans. ${ }^{7}$

The cystic fibrosis transmembrane conductance regulatory (CFTR) gene contains 27 exons and is localized on $7 \mathrm{q} 31$.

Corresponding author: Mehmet Köse, Division of Pediatric Pulmonology, Department of Pediatrics, Erciyes University, Kayseri, Turkey

e-mail: mhmtkose@yahoo.com

Received: March 31, 2021 Accepted: August 26, 2021 Available Online Date: September 29, 2021 • DOI: 10.5152/balkanmedj.2021.21199

Available at www.balkanmedicaljournal.org

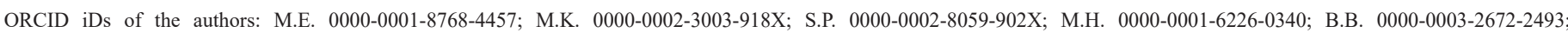

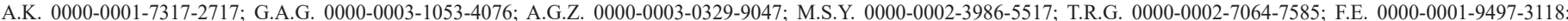
T.Ş.E. 0000-0001-7284-4999; A.T.A. 0000-0002-5360-8517

Cite this article as:

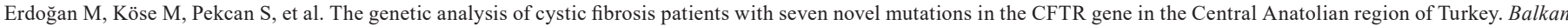
Med J.; 2021; 38(6):357-364.

Copyright@Author(s) - Available online at http://balkanmedicaljournal.org/ 
The protein is composed of 1480 amino acids. CFTR is an integral membrane protein that operates as a regulated chloride channel in the epithelia. Cystic fibrosis occurs due to homozygous or compound heterozygous pathogenic variants in the CFTR (CFTR/ $A B C C 7:$ MIM*602421) gene. Today, more than 2000 disease-causing variants of the CFTR gene have been found and their ethnic and geographical distributions show differences. ${ }^{8}$ The most common variant seen in almost all the studies is F508del. In addition, the frequency of more than 20 variants is more significant than $0.1 \%$ (www.genet.sickkid.on.ca/Home.html.).

A variety of CFTR mutations have a different effect on the CFTR protein. Therefore, the traditional classification of CFTR mutations consists of 6 groups. Class I: Nonsense, frameshift, or splice junction variants such as c. $1717-1 \mathrm{G}>\mathrm{A}, \mathrm{c} .1624 \mathrm{G}>\mathrm{T}$, and c. $3846 \mathrm{G}>\mathrm{A}$ can cause reduced or absent synthesis. Class II: Some missense variants and amino acid deletions such as c.1521_1523delCTT, c. $3909 \mathrm{C}>\mathrm{G}$, and c. $1682 \mathrm{C}>\mathrm{A}$ disrupt the traffic. Class III: Block the regulation of CFTR chloride channel (such as c.1652G $>\mathrm{A}$, c. $1645 \mathrm{~A}>\mathrm{C}, \mathrm{c} .4046 \mathrm{G}>\mathrm{A})$. Class IV: Alter conductance of the CFTR chloride channel (c.350G $>$ A, c.1000C $>$ T, c.1364C $>$ A). Class V: Some missense or splice site variants such as c.3272-26 A $>$ G can reduce the synthesis and cause less protein production. Class VI: Some mutations, such as c.120del123 may result in a less stable CFTR protein. ${ }^{9,10}$

The present study was conducted to describe the allele distribution and frequency of CFTR gene mutations, including rare variants, in patients diagnosed with $\mathrm{CF}$ in the Central Anatolian region of Turkey.

\section{MATERIAL AND METHODS}

\section{Study Populations}

The study was carried out in the Central Anatolian region in 3 pediatric pulmonology departments (Kayseri, Konya, and Ankara) in Turkey between July 2014 and December 2017. The patients were assessed in the Erciyes University School of Medicine, Kayseri Training and Research Hospital, Gazi University School of Medicine and Necmettin Erbakan University Meram Medicine Faculty. The Erciyes University Clinical Research Ethical Board was referred to for the study.

The diagnosis of $\mathrm{CF}$ was based on sweat chloride levels ( $>60 \mathrm{mEq} / \mathrm{L}$ ) and/or 2 identified CF mutations, as well as on characteristic symptoms of $\mathrm{CF}$ or a positive newborn screening test. ${ }^{11} \mathrm{Of}$ the total of 316 patients who were screened for genetic analysis, 215 patients who had homozygous or compound heterozygous mutations participated in the study. One hundred one patients (the variant was found only in one CFTR gene in 49 patients, no disease-causing mutation was found in 52 patients) were excluded from the study.

Genomic DNA isolation of the patients based on peripheral blood was made by using a DNA isolation kit according to the instructions of the manufacturer (Zinexts Life Science Corporation, Taiwan).

\section{Sanger Sequencing Analysis}

In 169 cases, genetic mutation analysis was conducted by Sanger sequencing. The GML SeqFinder Sequencing System CFTR FULL Gene Kit (GML AG Altendorf, Switzerland) was used for this process. Products of the polymerase chain reaction (PCR) were observed after gel electrophoresis, and these products were purified using the ExoSAP kit (Exo SAP PCR purification kit, UAB Corporation, Cleveland, OH, USA). The same products were sequenced with the BigDye Terminator version 3.1, according to the manufacturer's instructions. Later, they were purified with Sephadex. In the last stage, the products were analyzed on the Applied Biosystems ${ }^{\circledR}$ Sanger Sequencing 3500 Series Genetic Analyzers device, and bioinformatics analysis was conducted using the SeqScape software v2.6.

\section{Next Generation Sequencing Analysis}

Mutation analysis was conducted on 147 patients using Next Generation Sequencing (NGS). The NEXTflex Cystic Fibrosis Amplicon panel (BIO SCIENTIFIC, a PerkinElmer Company, Austin, TX, USA) kit was used for mutation analysis. This panel includes $10.4 \mathrm{~kb}, 28$ coding exons, 1 promoter region, and 3 deep intronic regions. Sixty-one pairs of primers provide the amplification and sequencing of all exons coding the CFTR locus. The total length of the related amplicons differ between 83 and $226 \mathrm{bp}$. The related reading areas of libraries and primary pad areas have an average size of 137-280 bp. The products were uploaded to the MiSeq Illumina device (Illumina, San Diego, California). The data obtained were analyzed with the Integrative Genomics Viewer software (version 2.3.98). Sequence annotation and variant calling were performed by the Genomize SEQ platform (https://seq.genomize. com).

\section{MLPA (Multiplex Ligation-dependent Probe Amplification) Analysis}

When no pathogenic/likely pathogenic allele was detected with Sanger Sequencing and NGS, Multiplex Ligation-dependent Probe Amplification (MLPA) analysis was performed for deletion/duplication in the CFTR gene according to the manufacturer's recommendations. DNA samples were denatured at $95^{\circ} \mathrm{C}, \mathrm{MRC}$ HOLLAND MLPA Probemix-P091 CFTR Probe was added and left for hybridization at $60^{\circ} \mathrm{C}$ for 17.5 hours, and was continued the next day with the ligation step. At this step, hybridized probe oligos were ligated with a ligation enzyme. After the PCR cycle was completed, bound probes were amplified with a PCR primer. After these steps were completed, the amplified DNA products were read by capillary electrophoresis in an Applied Biosystems ${ }^{\circledR}$ Sanger Sequencing 3500 Series Genetic Analyzer device. These data obtained from the device were analyzed in the Coffalyser software version v.140701.0000 (MRC-Holland, Amsterdam, the Netherlands), and CFTR (NM_000492.3) was accepted as a reference. This performs a quality check and calculates probe ratios.

\section{Interpretation of Results}

CFTR region-specific MLPA probes are allele copy numbers of 2 (normal), 0 (homozygous deletion), 1 (heterozygous deletion), 
3 (heterozygous duplication), and occasionally 4 (homozygous duplication or heterozygous triplication).

\section{Variant Classification}

The guidelines of the American College of Medical Genetics and Genomics and Association for Pathology, ${ }^{12}$ with the classification-benign, likely benign, uncertain significance, likely pathogenic, and pathogenic - were used to classify a variant. The pathogenicity of the novel variants was determined with the recommendation of these guidelines. There are 2 groups of criteria: (1) pathogenic/likely pathogenic and (2) benign/likely benign. Every pathogenic criterion is classified as: very strong, PVS1; strong, PS1-4; moderate, PM1-6; and supporting, PP1-5; and each benign criterion is classified as: stand-alone, BA1; strong, BS1-4; or supporting, BP1-7. Then, a class was chosen from the 5-tier system, as follows: Class 1, pathogenic; Class 2, likely pathogenic; Class 3, variant of uncertain significance (used if it did not meet one of the criteria/showed conflicting benign or pathogenic evidence); Class 4, benign; and Class 5, likely benign.

\section{Use of Databases}

Allele frequency is an important and striking indicator for benign/ pathogenic characterization of a variant in Mendelian disorders. The gnomad database (https:/gnomad. broadinstitute.org/) was used for the annotation of variant frequency. The CFTR mutation database (http://www.genet.sickkids.on.ca/) was also used for known variants. ClinVar (https://www.ncbi.nlm.nih.gov/c linvar) gave us an important clinical phenotype, Additionally, these databases were also used: http:/exac.broadinstitute.org; http://browser.1000genomes.org; http://www.ncbi.nlm.nih.gov/snp (dbSNP); http://www.omim.org (OMIM); http://www.hgmd.org; http://www.hgvs.org/dblist/dblist.html (HGVS); http://www.ncbi. nlm.nih.gov/genome; and http:/www.ncbi.nlm.nih.gov/refseq/rsg.

Several in silico computational methods were used to identify the possible degree of pathogenicity of CFTR variants: (a) Mutation taster (http://www.mutationtaster.org/); (b) Polyhen-2 (http:// genetics.bwh.harvard.edu/pph2/), used for missense alterations; (c) Human Splicing finder (http://umd.be/Redirect.html), used for slice site variants; (d) CADD, used for analyzing evolutionary conservation and allelic diversity; and (e) SIFT (http://sift.jcvi. org), which predicts whether an amino acid substitution affects protein function.

\section{RESULTS}

A molecular genetic analysis of 215 patients diagnosed with $\mathrm{CF}$ from the provinces of Ankara, Konya, and Kayseri in the Central Anatolian region of Turkey was performed. Of the patients, 105 were male, while 110 were female. The median age at diagnosis was 5 months (10 days-20 years). CFTR gene analysis was performed for 316 patients and 215 of them were genetically diagnosed with CF. Sixty-three different mutations were found. One hundred twenty-six patients $(39.8 \%)$ were homozygous for a variant and $89(27.8 \%)$ were compound heterozygous. In 49 patients, only 1 variant was found; no disease-causing mutation was found in 52 patients, and these were excluded from the study.

\section{Variant Spectrum}

A total of 63 different variants were found and the frequency of 20 of these was $>1 \%$. These variants are shown in Table 1 . In our study, 29 missense, 12 nonsense, 13 splice sites, 7 frameshifts, and 2 large del/dup mutations were found.

In 5 of the patients, 3 different variants were found at the same time. Three of these were found in 3 siblings from the same family (N1303K/c.1210-11T>G /E217G). While the N1303K variant was in transposition with the c.1210-11 T $>\mathrm{G}$ and E217G variants, the F508del variant was in transposition with the F1052V and Q2P (novel variant) in one patient, and the $\mathrm{R} 170 \mathrm{C}$ variant was in transposition with the F1052V and Q2P in another patient. MLPA analysis was conducted in patients with a mutation in one CFTR gene or who had no mutation. Exon 2 deletion was found in 5 alleles, while exon 22 duplication was found in 2 alleles. Exon 2 deletion was homozygous in one patient, with Y515X in 2 patients and with F508del in 1 patient. In 2 patients, exon 22 duplication was with F508del.

Seven of the 63 variants were novel, and they were defined in this study for the first time. The clinical and laboratory findings of the new variants are listed below and shown in Table 2.

The c.1393-1G $>\mathbf{T}$ splice site variant was found in intron 10. This variant was found in 1 case and it was in a patient who was referred with a complaint of growth retardation and chronic diarrhea at the age of 4 months, due to steatorrhea. The result of the sweat chloride test was $102 \mathrm{mmol} / \mathrm{L}$. The patient was from the Kayseri region in Central Anatolia.

The c.865_869delAGACA variant was found in exon 7. The sweat chloride test result was high $(74 \mathrm{mmol} / \mathrm{L})$ in the patient who referred with Pseudo Bartter syndrome (PBS) at 4 months. Bronchiectasis and diabetes developed in the follow-up. Pseudomonas and MRSA grew in the cultures of sputum. This novel variant was also in homozygous form and the patient came from the Konya region.

The c.2909-1G $>\mathbf{C}$ variant found in intron 18 in a patient who was from the Kayseri region. The patient had diarrhea, vomiting, and failure to thrive. PBS was developed in the follow-up. He had steatorrhea and a high sweat chloride test result $(93 \mathrm{mmol} / \mathrm{L})$. This variant was a homozygous mutant.

The c.5A $>\mathbf{C}$ variant was found in 2 patients. In the first of the 2 different cases, which were found to have c.5A $>$ C with F1052V, this novel variant was in transposition with the $\mathrm{R} 170 \mathrm{C}$ variant. In this patient, steatorrhea and bronchiectasis were found in the neonatal period. In the second case, the same variant was with F1052V and in transposition with F508del. This patient had a diagnosis of PBS, and the sweat chloride test result was high (74.1 $\mathrm{mmol} / \mathrm{L})$. 


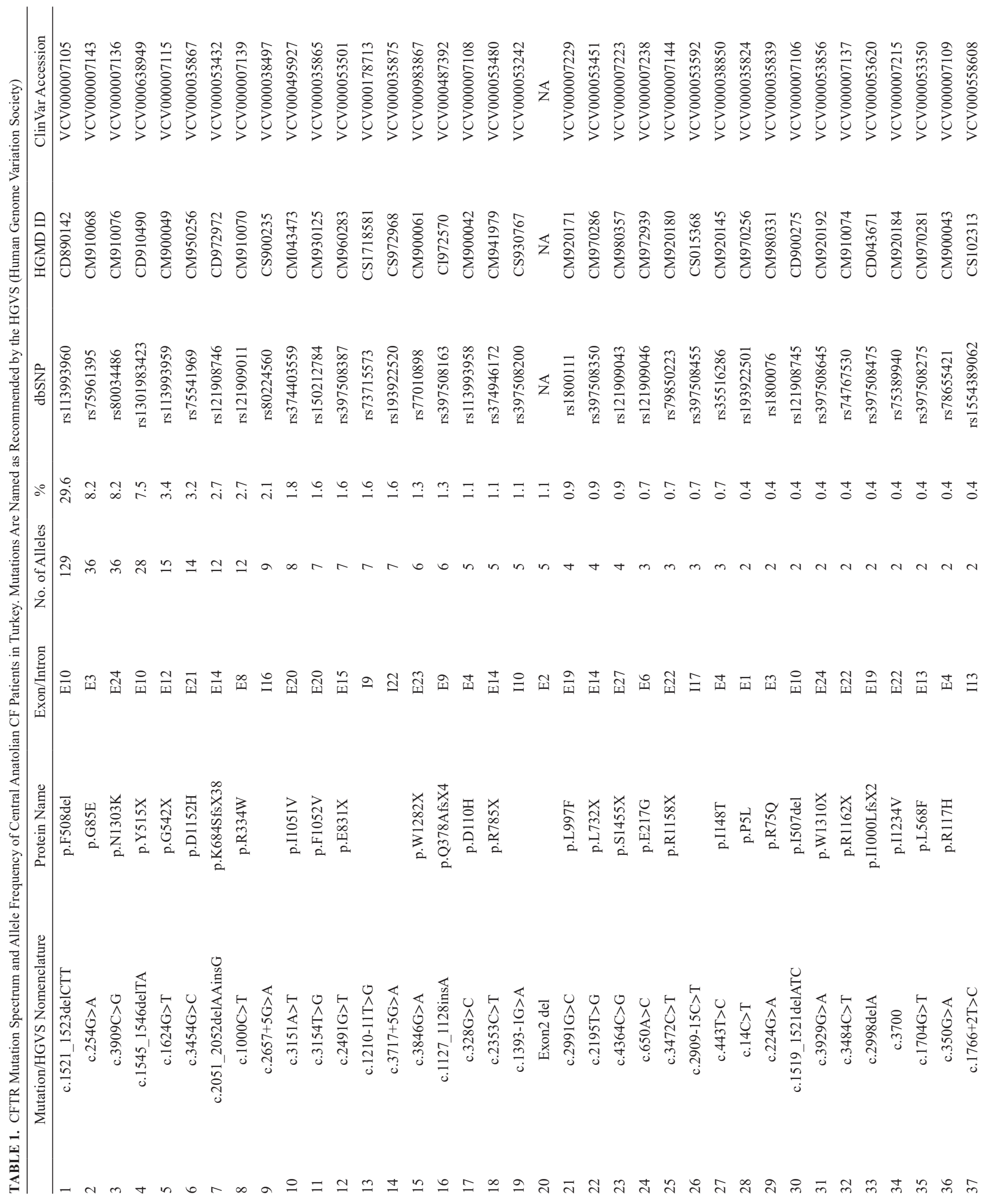



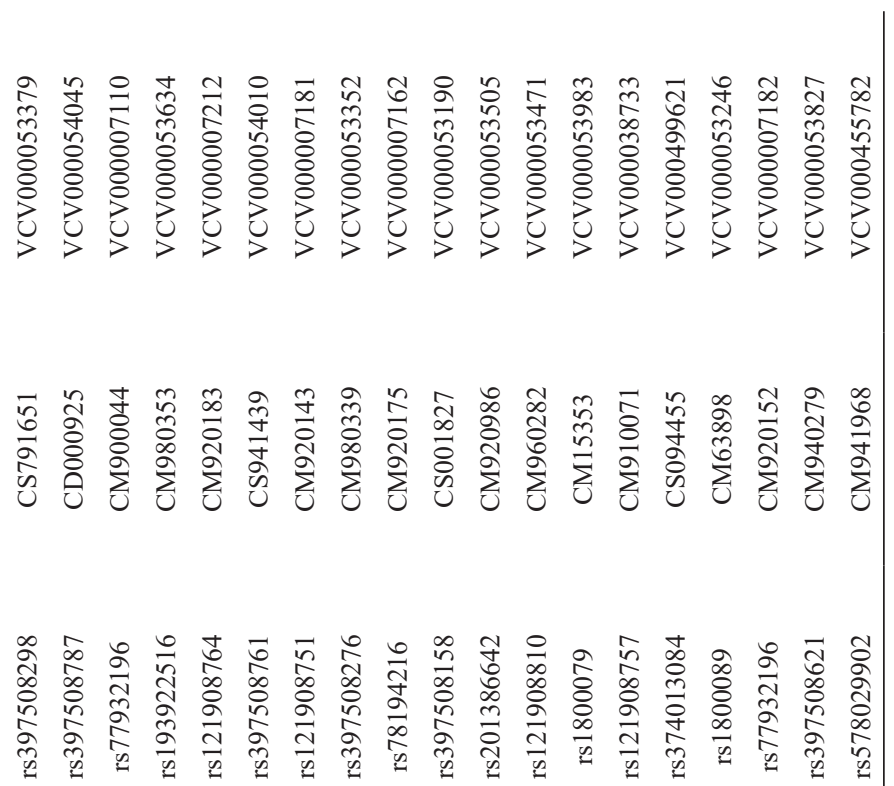

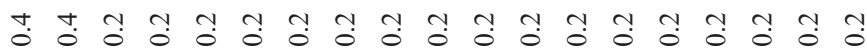

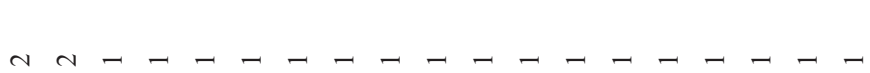

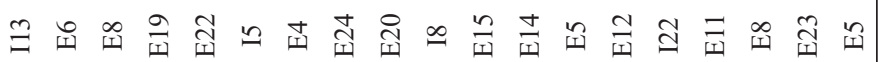
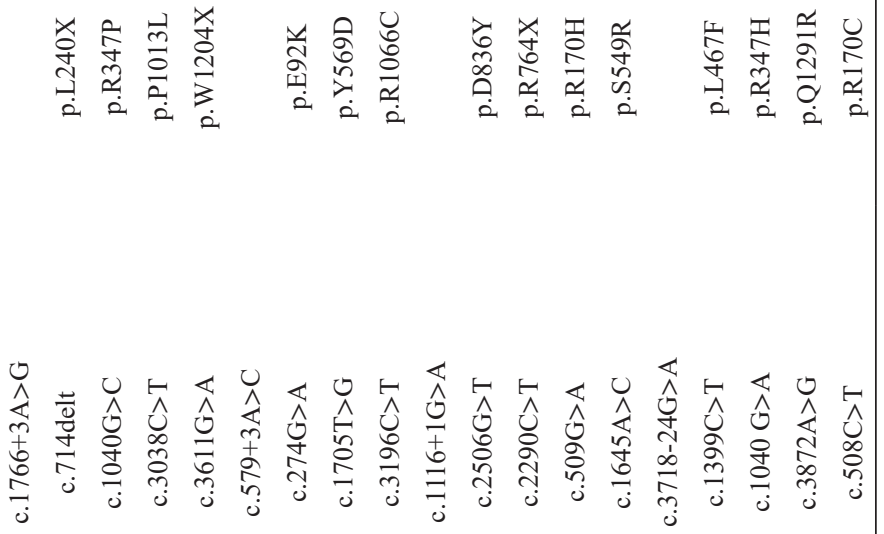

隽
The $\mathbf{c . 3 4 0 7 C}>\mathbf{A}$ variant was found in exon 21 and was in transposition with G85E. As in other patients, PBS was present, and the sweat chloride test result was high $(79 \mathrm{mmol} / \mathrm{L})$.

The $\mathbf{c} \mathbf{1 2 1 0 - 2} \mathbf{A}>\mathbf{G}$ variant was found in intron 16. This variant and the R347P variant were in transposition with F508del and the patient had PBS, with a high sweat chloride test result $(102 \mathrm{mmol} / \mathrm{L})$, and steatorrhea.

\section{Novel Duplication Exon 22}

A novel duplication was found in 2 patients who were siblings. The younger patient was diagnosed with PBS and steatorrhea; although the other sibling had PBS, the sweat chloride test was normal at first, while subsequent values were found to be high ( 84 and 86 $\mathrm{mmol} / \mathrm{L})$. No fat was detected in stool. In the genetic analyses of both siblings, there was heterozygous duplication in the whole exon 22 and at the same time, there was F508del in the other allele.

The $\mathrm{N} 1303 \mathrm{~K} / \mathrm{E} 217 \mathrm{G}$ and c.1210-11T $>\mathrm{G}$ variants were found in 3 family members. The first of these was found through neonatal screening. PBS, high sweat chloride test result, distal intestinal obstruction in advanced periods, and $S$. aureus colonization in the phlegm were found. These variants were found by CFTR molecular examinations carried out due to similar results in other siblings.

\section{DISCUSSION}

The present study provides an extensive review of the variants in the CF gene in a representative cohort in the Central Anatolian region of Turkey. As a result of the study, 63 different variations were found in $215 \mathrm{CF}$ patients. In our study, variant variation shows high heterogeneity in the gene. As can be seen in studies conducted, the Mediterranean region, including Turkey, is the region with the highest mutation heterogeneity, ${ }^{13-20}$ while 121 different variants were found in a study in the Spanish population, ${ }^{21}$ 105 variants in the French population, ${ }^{22}$ and 82 different variants in North-Eastern Italy. ${ }^{23}$ In contrast, relatively homogeneous groups are also found in this region. ${ }^{24}$ Genetically, the Anatolian population is very heterogeneous. Anatolia is a genetic bridge between the East and the West. In specific periods of history, these lands have hosted great migrations. This is thought to be the source of the high heterogeneity in the Turkish population.

The F508del variant was the most frequent in our study (29.6\%). This variant was found as homozygous in 40 patients and as compound heterozygous in 40 patients. While this rate is low, especially when compared with European countries, it complies with the geographical distribution-decreasing from the north to the south. . $^{17,18,21,25,26}$ The rates of F508del are similar to those in Turkey's southern and eastern neighbors, and higher than in Arab states. $^{20,27-31}$

In our study, c. 254G >Ap.(G85E) and c.3909C > G p.(N1303K) were the second most frequent variants $(8.2 \%)$. G85E was found in a total of $36 \mathrm{CF}$ chromosomes (homozygous in 13 cases and compound heterozygous in 10 cases). It was first defined by Zielenski et al..$^{32}$ This rate can be the highest among the studies conducted recently. The closest rate to this was found in a study conducted 


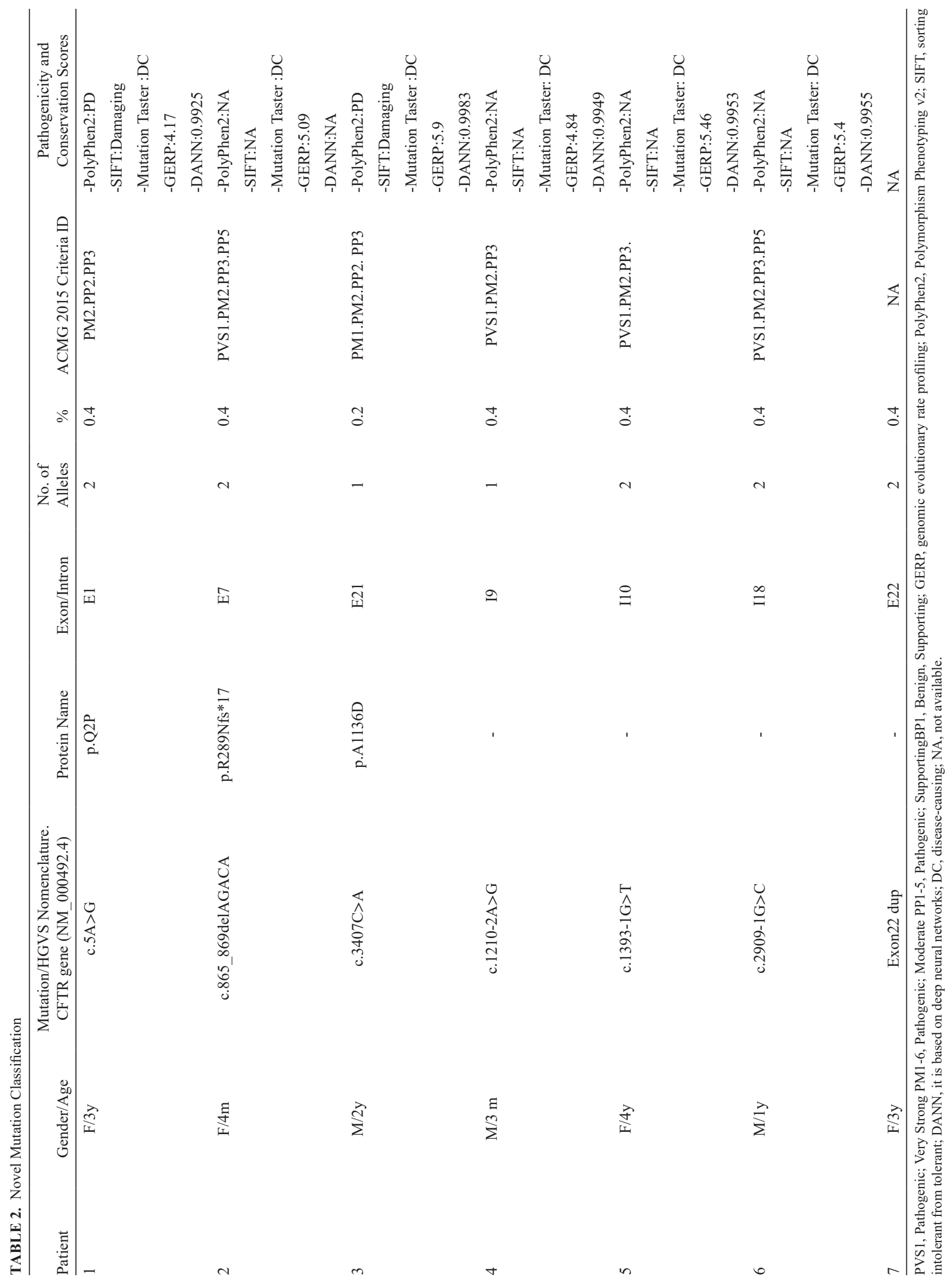


in Syria, a southern neighbor of Turkey; however, the patient population was quite limited. ${ }^{30}$ The relative frequency was found to be around $0.2 \%$ in studies conducted (www.genet.sickkid.on.ca/ Home.html). Although it is the second most frequent variant in our cohort, it was rare or nonexistent in previously conducted studies. ${ }^{13-16}$ This data can recall that carriers are more intense in the region of Turkey. Data from a more extensive and higher number of participants should be analyzed to determine whether there is geographical specificity to the G85E variant.

The N1303K variant is the second most common with the G85E, and its rate in the study population was $8.2 \%$. The relative frequency found in the studies conducted was $1.3 \%$ (www.genet.sickkid.on.ca/Home. html). In terms of frequency, the related variant has been reported as over $1 \%$ in many studies conducted, and it is among the most frequent of 5 variants. ${ }^{13-16}$ Although it differs in various ethnic groups and regions, it is the most prevalent in the Mediterranean region. The highest rates have been reported in studies conducted in Italian, Spanish, and Bulgarian populations. ${ }^{19,21,33}$ Although it was among the most frequently seen in previously conducted studies in Turkey, its frequency was found to be relatively low in our study. Unlike F508del, the variant frequency was found to increase in the northern-southern gradient in the European population.

The Y515X variant, the fourth most frequent variant in our study, is important since it is rare or nonexistent in other European communities and in the Mediterranean basin. It is among the most frequent variants in studies conducted in Turkey ${ }^{13,16}$ and the Turkish population in Bulgaria. ${ }^{19}$ The related study also reported that the haplotype of this variant was related to the same haplotype in the Bulgarian population, and that these results supported the hypothesis that the variant can have only one origin. This information was not confirmed since our study did not include haplotype analysis.

The G542X variant was the fifth most frequent variant found in the study $(3.4 \%)$. This variant is regular in the Mediterranean population. Its highest prevalence is in the Spanish population. ${ }^{21}$ It can be said that this population is distribution-specific for the Mediterranean geography. ${ }^{18,23,24,34}$ The frequency in our study was similar to the relative frequency $(2.6-2.4 \%)$.

The most apparent difference between our study and the studies in the Mediterranean region and Turkey is that the 2 variants with a frequency of over $1 \%$ were not found. ${ }^{18,21,23,24}$ These are c. $2183 \mathrm{AA}>\mathrm{G}$ and c. $2789+5 \mathrm{G}>\mathrm{A}$. Although they were most frequently seen in 3 other studies in Turkey, they were not found in our study. ${ }^{13,14,16}$ The reason for this should be the low carrier frequency of these variants in our study area.

Genetic heterogeneity is more frequent in European-origin communities. ${ }^{17,18,21,23,25}$ Communities other than those with a European origin (Middle East, Arabia, Far East) ${ }^{27-31}$ are weak in terms of CFTR alleles, with the Anatolian community as an exception. High heterogeneity should be expected in future Turkish population studies, as shown in the previous studies ${ }^{13-16}$ and in the present study. Our study shows the highest heterogeneity reported in Turkey so far. At the same time, while it shows partial similarities with previously reported studies in terms of frequent variants, some variants are reported in literature and databases as rare variants.

Among the 215 patients with homozygous or compound heterozygous mutations, 38 were eligible for elexacaftor/tezacaftor/ivacaftor therapy, 1 of them was eligible for ivacaftor therapy, 17 were eligible for both tezacaftor/ivacaftor and ivacaftor therapy, 26 were eligible for elexacaftor/tezacaftor/ivacaftor, ivacaftor and lumacaftor/ivacaftor therapy, 16 were eligible for tezacaftor/ivacaftor, elexacafto r/tezacaftor/ivacaftor, and lumacaftor/ivacaftor therapy, 3 of them were eligible for elexacaftor/tezacaftor/ivacaftor, ivacaftor, and tezacaftor/ivacaftor therapy, 1 patient was eligible for both lumacaftor/ivacaftor and tezacaftor/ivacaftor therapy, and 1 patient was eligible for ivacaftor and elexacaftor/tezacaftor/ivacaftor therapy. Approximately $47.9 \%$ of the study population was eligible for modulatory drug therapy. This rate was lower than that of the North American and European communities and might be due to the lower incidence of the F508del variant in the study population.

This study has some limitations. Although we analyzed $\pm 50 \mathrm{bp}$ to all coding regions of the CFTR gene and exon-intron boundaries, deep intronic CFTR mutations that may cause CF could not be analyzed for technical reasons. In addition, in some of the patients in whom we did not find any mutations or in whom only heterozygous pathogenic/likely pathogenic mutations were detected in the CFTR gene, we could not perform the MLPA test because they did not continue to patient follow-up. Another limitation of the study is the absence of consanguineous marriage rates.

In conclusion, the present study contributes to a better understanding of the heterogeneous structure of CFTR mutations in the Turkish population. Contrary to what is known, we report that the $\mathrm{CF}$ disease is observed in a high frequency and broad spectrum outside Europe and especially in Turkey.

Ethics Committee Approval: Ethical committee approval was received from the Ethics Committee of Erciyes University School of Medicine, (2016/355).

Patient Consent for Publication: Informed consent was obtained from all patients

Data-sharing Statement: The data that support the findings of this study are available from the corresponding author upon reasonable request.

Author Contributions: Concept - M.E., M.K., S.P., M.H., A.T.A.; Design - M.E., M.K., M.H.; Supervision - M.E., M.K., S.P., M.H., A.T.A.; Materials - M.E., M.K., S.P., M.H., B.B., A.K., G.A.G., A.G.Z., M.S.Y., T.R.G., F.E., T.Ş.E., A.T.A.; Data Collection and/or Processing - M.E., M.K., S.P., M.H., B.B., A.K., G.A.G., A.G.Z., M.S.Y., T.R.G., F.E., T.Ş.E., A.T.A.; Analysis and/or Interpretation - M.E., M.K., S.P., M.H., B.B., A.K., G.A.G., A.G.Z., M.S.Y., T.R.G., F.E., T.Ş.E., A.T.A.; Literature Review - M.E., M.K.; Writing M.E., M.K.; Critical Review - M.E., M.K., S.P., M.H., B.B., A.K., G.A.G., A.G.Z., M.S.Y., T.R.G., F.E., T.Ş.E., A.T.A.

Conflict of Interest: The authors have no conflicts of interest to declare.

Funding: The authors declared that this study had received no financial support.

\section{REFERENCES}

1. Salvatore D, Buzzetti R, Baldo E, et al. An overview of international literature from cystic fibrosis registries. Part 3. Disease incidence, genotype/phenotype correlation, microbiology, pregnancy, clinical complications, lung transplantation, and miscellanea. J Cyst Fibros. 2011;10(2):71-85. [CrossRef] 
2. Southern KW, Munck A, Pollitt R, et al. A survey of newborn screening for cystic fibrosis in Europe. J Cyst Fibros. 2007;6(1):57-65. [CrossRef]

3. Mall MA, Hartl D. CFTR: cystic fibrosis and beyond. Eur Respir J. 2014;44(4):10421054. [CrossRef]

4. Hangül M, Pekcan S, Kose M, et al. The incidence of cysticfibrosis in Central Anatolia region of Turkey in 2015 and 2016. Balkan Med J. 2019;36:179-183.

5. Grody WW, Cutting GR, Klinger KW, et al. Subcommittee on Cystic Fibrosis Screening, Accreditation of Genetic Services Committee, ACMG. Laboratory standards and guidelines for population-based cystic fibrosis carrier screening. Genet Med. 2001;3(2):149-154. [CrossRef]

6. Watson MS, Cutting GR, Desnick RJ, et al. Cystic fibrosis population carrier screening: 2004 revision of American College of Medical Genetics mutation panel. Genet Med. 2004;6(5):387-391. [CrossRef]

7. Schrijver I, Oitmaa E, Metspalu A, Gardner P. Genotyping microarray for the detection of more than 200 CFTR mutations in ethnically diverse populations. J Mol Diagn. 2005;7(3):375-387. [CrossRef]

8. Shrimpton AE. Molecular diagnosis of cystic fibrosis. Expert Rev Mol Diagn. 2002;2(3):240-256. [CrossRef]

9. De Boeck K, Amaral MD. Progress in therapies for cystic fibrosis. Lancet Respir Med. 2016;4(8):662-674. [CrossRef]

10. Marson FAL, Bertuzzo CS, Ribeiro JD. Classification of CFTR mutation classes. Lancet Respir Med. 2016;4(8):e37-e38. [CrossRef]

11. Kose M, Pekcan S, Kiper N, et al. Doll-like face: is it an under estimated clinical presentation of cystic fibrosis? Pediatr Pulmonol. 2008;43(7):634-637. [CrossRef]

12. Richards S, Aziz N, Bale S, et al. Standards and guidelines for the interpretation of sequence variants: a joint consensus recommendation of the American College of Medical Genetics and Genomics and the Association for Molecular Pathology. Genet Med. 2015;17(5):405-424. [CrossRef]

13. Onay T, Zielenski J, Topaloglu O, et al. Cystic fibrosis mutations and associated haplotypes in Turkish cystic fibrosis patients. Hum Biol. 2001;73(2):191-203. [CrossRef]

14. Kilinç MO, Ninis VN, Dağli E, et al. Highest heterogeneity for cystic fibrosis: 36 mutations account for $75 \%$ of all CF chromosomes in Turkish patients. Am J Med Genet. 2002;113(3):250-257. [CrossRef]

15. Onay T, Topaloglu O, Zielenski J, et al. Analysis of the CFTR gene in Turkish cystic fibrosis patients: identification of three novel mutations (3172delAC, P1013L and M1028I). Hum Genet. 1998;102(2):224-230. [CrossRef]

16. Dogru D, Cakır E, Şişmanlar T, et al. Cystic fibrosis in Turkey: first data from the national registry. Pediatr Pulmonol. 2020;55(2):541-548. [CrossRef]

17. Dörk T, Mekus F, Schmidt K, et al. Detection of more than 50 different CFTR mutations in a large group of German cystic fibrosis patients. Hum Genet. 1994;94(5):533542. [CrossRef]

18. Kanavakis E, Efthymiadou A, Strofalis S, et al. Cystic fibrosis in Greece: molecular diagnosis, haplotypes, prenatal diagnosis and carrier identification amongst high-risk individuals. Clin Genet. 2003;63(5):400-409. [CrossRef]
19. Angelicheva D, Calafell F, Savov A, et al. Cystic fibrosis mutations and associated haplotypes in Bulgaria-comparative population genetic study. Hum Genet. 1997 ;99(4):513-520. [CrossRef]

20. Alibakhshi R, Kianishirazi R, Cassiman JJ, Zamani M, Cuppens H. Analysis of the CFTR gene in Iranian cystic fibrosis patients: identification of eight novel mutations. J Cyst Fibros. 2008;7(2):102-109. [CrossRef]

21. Alonso MJ, Heine-Suñer D, Calvo M, et al. Spectrum of mutations in the CFTR gene in cystic fibrosis patients of Spanish ancestry. Ann Hum Genet. 2007;71(2):194-201. [CrossRef]

22. Chevalier-Porst F, Bonardot AM, Gilly R, et al. Mutation analysis in 600 French cystic fibrosis patients. J Med Genet. 1994;31(7):541-544. [CrossRef]

23. Gasparini P, Marigo C, Bisceglia G, et al. Screening of 62 mutations in a cohort of cystic fibrosis patients from North Eastern Italy: their incidence and clinical features of defined genotypes. Hum Mutat. 1993;2(5):389-394. [CrossRef]

24. Quint A, Lerer I, Sagi M, Abeliovich D. Mutation spectrum in Jewish cystic fibrosis patients in Israel: implication to carrier screening. Am J Med Genet A. 2005;136(3):246248. [CrossRef]

25. Schwartz M. Cystic fibrosis transmembrane conductance regulator (CFTR) gene: mutations and clinical phenotypes. Ugeskr Laeger. 2003;165(9):912-916.

26. Ziętkiewicz E, Rutkiewicz E, Pogorzelski A, et al. CFTR mutations spectrum and efficiency of molecular diagnostic in Polish cystic fibrosis patients. PLoS One. 2014;9(2):e89094. [CrossRef]

27. Jarjour RA, Al-Berrawi S, Ammar S, Majdalawi R. Spectrum of cystic fibrosis mutations in Syrian patients. Minerva Pediatr. 2018;70(2):159-164. [CrossRef]

28. Rawashdeh M, Manal H. Cystic fibrosis in Arabs: a prototype from Jordan. Ann Trop Paediatr. 2000;20(4):283-286. [CrossRef]

29. Banjar H. Geographic distribution of cystic fibrosis transmembrane regulator gene mutations in Saudi Arabia. East Mediterr Health J. 1999;5(6):1230-1235. [CrossRef]

30. El-Harith EA, Dörk T, Stuhrmann M, et al. Novel and characteristic CFTR mutations in Saudi Arab children with severe cystic fibrosis. J Med Genet. 1997;34(12):996-999. [CrossRef]

31. Eskandarani HA. Cystic fibrosis transmembrane regulator gene mutation in Bahrain. J Trop Pediatr. 2001;47:110-112.

32. Zielenski J, Bozon D, Kerem B, et al. Identification of mutations in exons 1 through 8 of the cystic fibrosis transmembrane conductance regulator (CFTR) gene. Genomics. 1991;10(1):229-235. [CrossRef]

33. Giordani B, Amato A, Majo F, et al. Gruppo di lavoro RIFC. Italian Cystic Fibrosis Registry Report. 2011-2014. Epidemiol Prev. 2018;42(1S1):1-32. [CrossRef]

34. Yalçıntepe S, Gürkan H, Atlı E, Sayın NC, Başaran ÜN. Two cases of cystic fibrosis with compound heterozygous variants reported for the first time. Balk Med J. 2020;37(5):297-298. [CrossRef] 